\title{
Management of giant intermuscular lipoma of hips: A case report and review of literature
}

\author{
DA-REN LIU* ${ }^{*}$ CHAO LI* and LI CHEN \\ Department of Surgery, Second Affiliated Hospital, Zhejiang University School of Medicine, Hangzhou 310009, P.R. China
}

Received July 16, 2012; Accepted December 5, 2012

DOI: $10.3892 / \mathrm{mco} .2013 .63$

\begin{abstract}
Intermuscular lipomas are rare soft tissue tumors of mesenchymal origin. They are closely correlated with other benign and malign mesenchymal tumors in clinical presentation and imaging, causing problems in diagnosis and treatment. This study examined a rare giant intermuscular lipoma of the hip and its management, allowing clinicians to become familiar with this lesion. Furthermore, the clinical data of 13 patients with pathologically confirmed giant intermuscular lipomas ( 1 case treated by the authors, the remaining 12 cases reported in the Chinese literature during the period from January, 1992 to May, 2012) were also reviewed and analyzed. Based on the review, intermuscular lipomas were found to be more common in males and usually presented with an asymptomatic mass, with an average patient age of 39.7 years (range, 11-70), while symptom duration was between 1 month and 10 years. The most common tumor origin sites were the thigh and forearm and the option of treatment was surgical excision. The present study showed that it is crucial for the surgeon to consider infiltrating lipomas when dealing with deep soft tissue masses, while complete removal of the lesion was vital to avoid recurrence.
\end{abstract}

\section{Introduction}

Lipomas are the most common soft tissue tumors with a prevalence rate of 2.1 per 1,000 people (1). Lipomas are benign tumors of mesenchymal origin composed of mature lipocytes (2) and may be localized in any region of the body, superficial or deep (3). Generally, lipomas are subcutaneous, small, multiple and weigh only a few grams (4), their preferable locations being the thigh, shoulder and trunk (5). However, a small number of lipomas may also be subfascial and further

Correspondence to: Professor Li Chen, Department of Surgery, Second Affiliated Hospital, Zhejiang University School of Medicine, 88 Jiefang, Zhejiang, Hangzhou 310009, P.R. China

E-mail: chenli@mail.hz.zj.cn

${ }^{*}$ Contributed equally

Key words: lipoma, intermuscular, management classified as parosteal, interosseous or visceral, as well as infiltrating lipomas (6) [including inter- and intramuscular lipomas, with an estimated incidence of 1.8 and $0.3 \%$, respectively (7)]. Intermuscular lipomas are thought to arise from the intermuscular septa and to enlarge between muscle bundles, while the lesions are usually well-circumscribed and easily separated during surgery. Intramuscular lipomas, however, arise between muscle fibers, pass through the intermuscular septa and infiltrate the surrounding tissues, rendering removal of the lesion from the nearby muscles difficult (8-10). Liposarcomas are malignant soft tissue tumors reported in radiological as well as histological findings to comprise 7-27\% of the soft tissue sarcomas (11), and be able to mimic inter- and intramuscular lipomas, rendering the diagnosis and choice of treatment difficult (12).

The aim of this study was to report a giant deep-seated intermuscular lipoma of the hip, and discuss the epidemiology, histopatholgy, imaging characteristics, differential diagnosis and management of the intermuscular lipomas.

\section{Materials and methods}

Identification of patients with intermuscular lipoma. The Chinese Biological and Medicine Database (between January, 1992 and May, 2012), the China Hospital Knowledge Database and the Chinese Science and Technology Periodical Database were searched. An additional search was carried out regarding the Chinese journals that were not included in the network database and 4 patients were identified. Duplicate reports were identified and excluded from further analysis to avoid overrepresentation. Twelve cases reported in China were identified. Informed consent was obtained from the patients and the study was approved by the Research Ethics Committee of the Zhejiang University.

Case report. A 50-year-old female patient was treated in our outpatient clinic with a complaint of post-traumatic swelling of the left hip. The patient had a history of a car accident and an injury of the left hip. She did not seek for medical treatment until she felt a swollen mass 20 days later. Physical examination showed a 12x12 cm lump located in the left hip, on the post-lateral side of the greater trochanter of the femur bone. The lump was smooth-surfaced, immobile, had increased skin tension and was non-pulsatile with mild tenderness, which radiated towards the lower limb. The laboratory examinations 
A

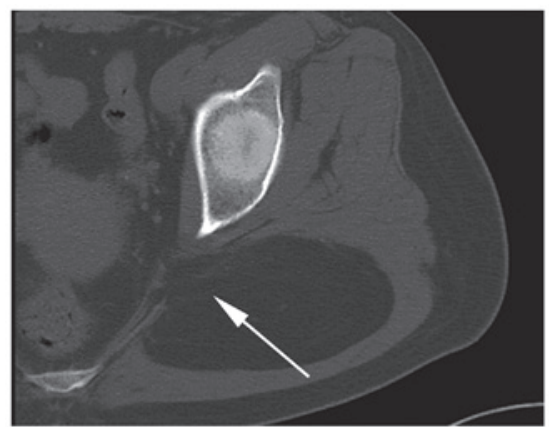

B

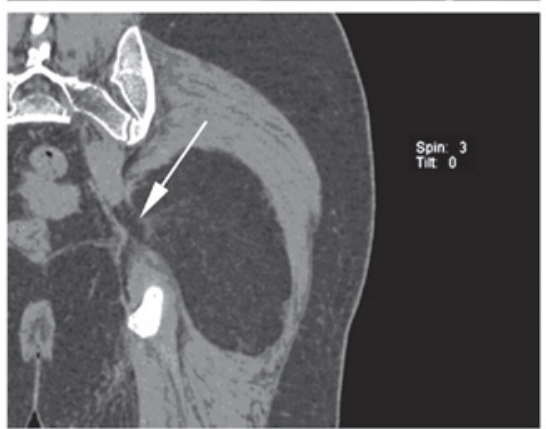

Figure 1. Intermuscular lipoma from the hip of a 50-year-old woman (A) Axial and (B) sagittal CT imaging revealed a fat density mass containing thin streaky densities under the gluteus maximus, with suspected infiltration of anteromedial muscle (arrow).

A

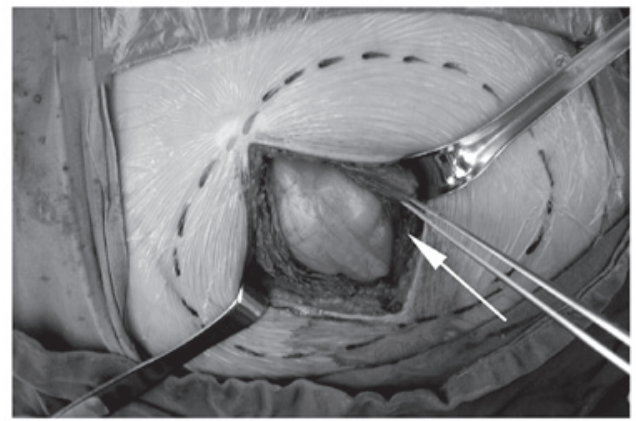

B

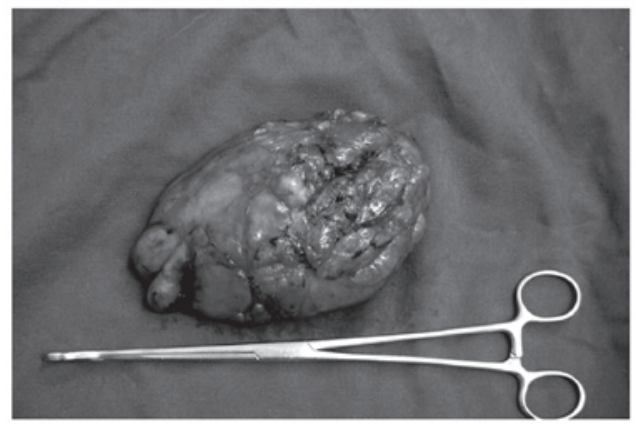

Figure 2. Gross view of the intermuscular lipoma. (A) Intraoperative view shows that the lesion was located under the gluteus maximus (arrow). (B) The dissected intermuscular lipoma was $10 \times 13 \times 6 \mathrm{~cm}$ in size.

were normal. The ultrasonographic examination showed a $5.0-\mathrm{cm}$ liquid dark area deep in the muscular layer, with tiny high-level echo spots floating inside. No significant blood flow signals were detected by color Doppler flow imaging (CDFI). Based on the present history, clinical presentations and ultrasonographic outcome, the patient was admitted for

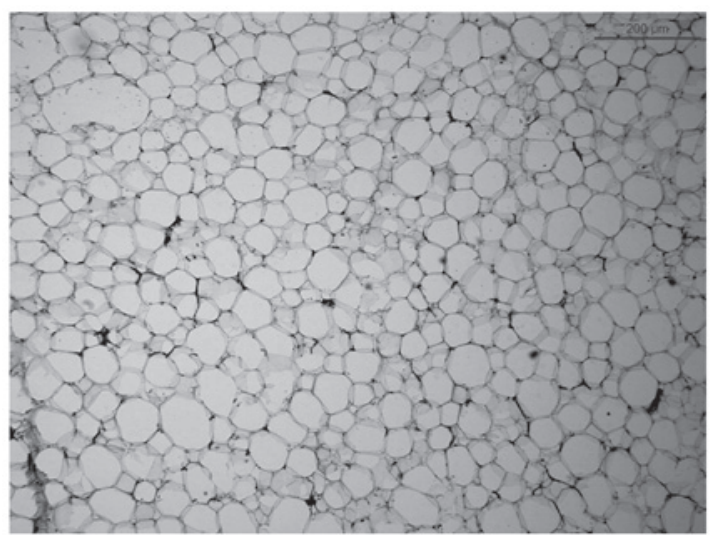

Figure 3. Histological examination findings of the tumor. The lesion comprised mature univacuolated lipocytes of a fairly uniform size, without any involvement of muscle fibers. No cellular atypia, mitosis or necrosis was observed (hematoxylin and eosin; magnification, x200).

post-traumatic hematoma. However, further examination of computed tomography (CT) demonstrated a $10.4 \times 5.3 \times 13-\mathrm{cm}$ diameter fat density mass [CT value-95 Hounsfield Units (HU)], accompanied by slightly higher density streaky structures with obscure boundaries under the left gluteus maximus, possibly infiltrating the anteromedial muscle (Fig. 1).

During surgery, an incision parallel with the muscle fiber of the gluteus maximus was selected. A circumscribed mass with intact capsule was noted beneath the gluteus maximus, compressing the surrounding muscles (the gluteus maximus and piriformis muscle), and partly protruding through the infrapiriform foramen, although no vessels or nerves were involved (Fig. 2). The mass was removed en bloc, while protecting the sciatic nerve.

The histopathologic examination showed a giant intermuscular lipoma of the left hip (10x13x6 cm), comprising mature fat cells without the involvement of muscle fibers, with no evidence of cellular atypia, mitosis or necrosis (Fig. 3). The patient recovered and was discharged 3 days after surgery without any complications. A 6-month follow-up was carried out and no recurrence was detected.

\section{Results}

Twelve reported cases of intermuscular lipoma, surgically treated in China between January, 1992 and May, 2012 were retrospectively reviewed. The detailed information is shown in Table I. There were 8 (66.7\%) males and 4 females $(33.3 \%)$, indicating that intermuscular lipoma occurred more frequently in men, which was consistent with the findings reported in the study by Nishida et al (12). The patients had an average age of 39.7 years (range, 11-70), with a symptom duration between 1 month and 10 years (mean, 41.8 months). The most common site of tumor origin was the thigh (6 patients, $50 \%$ ), followed by the forearm ( 2 patients, $16.7 \%$ ). Additional sites, such as the neck, submental, buttock and midpalmar space were also reported. As for symptoms, an asymptomatic mass was the most frequent presentation (6 patients, 50\%), followed by swelling mass (4 patients, 33.3\%). Movement disorder and numbness were also reported, possibly generated by compres- 
Table I. Characteristics of intermuscular lipomas reported in China during the past 20 years.

\begin{tabular}{|c|c|c|c|c|c|c|}
\hline Patient & Gender & Age (years) & Duration of symptoms & Dimensions $(\mathrm{cm})$ & Localization & Symptoms \\
\hline 1 & M & 45 & 3 months & $5 \times 3 \times 1.5$ & Forearm & Movement disorder \\
\hline 2 & M & 46 & 6 years & $15 \times 10 \times 8$ & Neck & Painless mass \\
\hline 3 & M & 11 & 5 years & $1.5 \times 3.0$ & Submental & Painless mass \\
\hline 4 & $\mathrm{~F}$ & 51 & 2 months & $15 \times 15 \times 6$ & Buttock & Painless mass \\
\hline 5 & $\mathrm{~F}$ & 40 & 4 years & $14 \times 6.5 \times 8$ & Thigh & Numbness and mass \\
\hline 6 & $\mathrm{~F}$ & 52 & 1 month & $25 \times 10 \times 8$ & Thigh & Swelling and mass \\
\hline 7 & M & 70 & 10 years & $6 \times 5$ & Forearm & Painless mass \\
\hline 8 & M & 26 & 2 years & $14.6 \times 13.5 \times 6.1$ & Thigh & Swelling and mass \\
\hline 9 & M & 20 & 6 months & $13 \times 13 \times 6$ & Thigh & Painless mass \\
\hline 10 & M & 60 & 5 years & $29 \times 20 \times 3.5$ & Thigh & Swelling and mass \\
\hline 11 & M & 29 & 9 months & $20 \times 16 \times 7$ & Thigh & Swelling and mass \\
\hline 12 & $\mathrm{~F}$ & 26 & 8 years & $5 \times 5 \times 2$ & Midpalmar space & Painless mass \\
\hline
\end{tabular}

M, male; F, female.

sion of the tumor to the surrounding nerves. Moreover, the clinical presentations were not closely correlated with the tumor size. For instance, patient 1 on the list had a small-sized lipoma $(5 \times 3 \times 1.5 \mathrm{~cm})$ that generated movement disorder, while patient 10 had the largest lesion reviewed, with no neuromuscular dysfunctions. Thus, a critically located lesion may induce a mass effect regardless of its size. The 12 patients underwent surgery following admission and recovered successfully.

\section{Discussion}

Lipomas are the most common benign soft tissue tumors that may occur anywhere in the body, and are mostly found within the subcutaneous areas. However, compared to common intermuscular lipomas, giant, deep-seated lipomas of the hip are even more infrequent and thus easily misdiagnosed. In this study, we presented an adult female patient with an unusually large, deep intermuscular lipoma in the left hip that led the initial misdiagnosis.

Due to the rare location, intermuscular giant lipomas are easily overlooked when making primary diagnosis upon admission. To differentiate from other soft tissue tumors, auxiliary examinations including ultrasonography, CT and magnetic resonance imaging (MRI) are needed, which may improve the diagnosis, as well as an appropriate staging of the tumor extension and involved structures. Ultrasonography of the lipomas is often the initial diagnostic procedure due to its availability and cost-effectiveness, when compared with CT and MRI. Generally, lipomas have been described as being homogeneous in echotexture and typically slightly hyperechoic to subcutaneous fat (13), although exceptions have frequently been observed (14). This may be caused by different compositions of the tumor since pure fatty tumors have less acoustic impedance due to fewer interfaces compared with tumors with a mixed composition of fat and water (15). Ultrasonography alone is not definitive, thus CT and MRI should be considered to further assess the nature of the lesion. Fat has low attenuation on CT, i.e., less than $-20 \mathrm{HU}$ and typically between
-65 and $-120 \mathrm{HU}$ (-95 HU in the present case) (16). As for intermuscular lipomas, CT shows a fat density mass, usually accompanying thin streaky densities (66.7\% reported), which are fibrous tissues of the intermuscular space. The thickness of these streaks is uniform and they are usually uninterrupted (12). On the MRI, intermuscular lipomas show a fat signal intensity mass similar to CT on T1- and T2-weighted images, although the thin streaky densities are less distinctive on MRI compared with the CT $(12,16)$.

Giant intermuscular lipomas should be differentiated from liposarcomas, malignant fibrous histiocytomas, metastatic carcinomas or other benign soft-tissue lesions, such as a cyst, hematoma, muscle herniation, cystic hygroma or fibrous myositis $(8,17)$. Intermuscular lipomas should also be distinguished from liposarcomas in terms of malignancy. The possibility of liposarcoma should be considered when a fatty tumor with a dimension of $>10 \mathrm{~cm}$ has shown rapid growth (11). Imaging examinations may be of crucial importance in the differentiation between the two tumors. On CT, a liposarcoma shows a fat density mass with areas of unclear amorphous density, usually accompanied by thick and thin streaky soft tissue densities, with occasionally interrupted streaks. On MRI, signal intensity of fat is evident, however, the intensity is lower compared with normal fat in certain areas, and the thick streaky structures are less distinctive when compared with the CT (12). Moreover, despite a proven negative imaging, a liposarcoma may still be malignant. Therefore, careful histopathologic evaluation is required, and the presence of nuclear pleomorphism and multinuclear giant cells may help to distinguish lipomas from malignant liposarcomas (18).

In the present case, the patient with post-traumatic hematoma was diagnosed initially based on her clinical symptoms (swelling for 20 days), trauma history and ultrasonography (liquid dark area in the muscular layer and no blood signal evident). Deep-seated lipomas should always be taken into consideration when dealing with soft tissue masses. The mechanism for intermuscular lipomas remains unclear, and trauma was reported as a related factor $(4,19,20)$. It was suggested that 
after a blunt trauma, rupture of the fibrous septa, which prevent the migration of fat, accompanied by tears of the anchorage between the skin and the deep fascia likely resulted in the proliferation of adipose tissue (20). However, in the present patient, the tumor was believed to have already existed prior to the accident and became evident after being extruded from the surrounding tissues by the trauma, given the limited time period between the trauma and mass formation (20 days).

The treatment for this type of tumor is complete excision. However, indications of the surgery may be discomfort by fascial compartment syndrome, the necessity of differential diagnosis from malignant tumors and the possible development of malignant alterations. Generally, intermuscular lipomas have a well-defined pseudo-capsule $(3,12,21)$, thus, dissection around these benign neoplasms is not particularly difficult. The surgical removal of the tumor ought to be complete since residual disease may lead to recurrence and additional surgical procedures with added risk and complications (22). Therefore, a thorough anatomic knowledge and experience must be obtained prior to attempting an excision. In the surgical operation, however, the extent of the resection is sometimes modified to avoid injuring important surrounding neurovascular or muscular tissues and causing functional impairment. This compromise between adequate surgical margins and functional disability may lead to incomplete resection and an obviously higher local recurrence rate. Following excision, the recurrence rate of intermuscular lipoma was described as modest, with the lowest rate at $3 \%$ and the highest at $62.5 \%$ (23), which was most likely due to incomplete surgical excision. Thus, the possibility of recurrence after long lipoma-free intervals are probable, while an extended period of long-term follow-up is mandatory.

In conclusion, intermuscular lipomas are rare benign tumors, with giant lesions being even more infrequent, and are generally presented with painless mass or swelling for years. It is important for clinicians to be aware of the infiltrating lipomas when dealing with deep-seated soft tissue masses. To make an accurate diagnosis, imaging techniques (ultrasonography, CT and MRI) may be helpful and useful, particularly when making differential diagnosis. Surgical removal is the treatment of choice providing a definite diagnosis, while a complete resection of the lesions is vital to avoid recurrences.

\section{References}

1. Silistreli OK, Durmus EU, Ulusal BG, Oztan Y and Gorgu M: What should be the treatment modality in giant cutaneous lipomas? Review of the literature and report of 4 cases. Br J Plast Surg 58: 394-398, 2005
2. Bispo Junior RZ and Guedes AV: Parosteal lipoma of the femur with hyperostosis: case report and literature review. Clinics (Sao Paulo) 62: 647-652, 2007.

3. Hakim E, Kolander Y, Meller Y, Moses M and Sagi A: Gigantic lipomas. Plast Reconstr Surg 94: 369-371, 1994.

4. Terzioglu A, Tuncali D, Yuksel A, Bingul F and Aslan G: Giant lipomas: a series of 12 consecutive cases and a giant liposarcoma of the thigh. Dermatol Surg 30: 463-467, 2004.

5. Copcu E, Sivrioglu N and Culhaci N: Axillary giant lipoma. Plast Reconstr Surg 114: 1982-1983, 2004.

6. Gonzalez-Crussi F, Enneking WF and Arean VM: Infiltrating angiolipoma. J Bone Joint Surg Am 48: 1111-1124, 1966.

7. Fletcher CD and Martin-Bates E: Intramuscular and intermuscular lipoma: neglected diagnoses. Histopathology 12: 275-287, 1988.

8. Pelissier A, Sawaf MH and Shabana AH: Infiltrating (intramuscular) benign lipoma of the head and neck. J Oral Maxillofac Surg 49: 1231-1236, 1991.

9. Lerosey Y, Choussy O, Gruyer X, et al: Infiltrating lipoma of the head and neck: a report of one pediatric case. Int J Pediatr Otorhinolaryngol 47: 91-95, 1999.

10. Ozcan C, Gorur K, Talas D and Aydin O: Intramuscular benign lipoma of the sternocleidomastoid muscle: a rare cause of neck mass. Eur Arch Otorhinolaryngol 262: 148-150, 2005.

11. Celik C,Karakousis CP,Moore Rand HolyokeED:Liposarcomas: prognosis and management. J Surg Oncol 14: 245-249, 1980.

12. Nishida J, Morita T, Ogose A, et al: Imaging characteristics of deep-seated lipomatous tumors: intramuscular lipoma, intermuscular lipoma, and lipoma-like liposarcoma. J Orthop Sci 12: 533-541, 2007.

13. Lin J, Jacobson JA, Fessell DP, Weadock WJ and Hayes CW: An illustrated tutorial of musculoskeletal sonography: part 4, musculoskeletal masses, sonographically guided interventions, and miscellaneous topics. AJR Am J Roentgenol 175: 1711-1719, 2000.

14. Volta A, Bonazzi M, Gnudi G, Gazzola M and Bertoni G: Ultrasonographic features of canine lipomas. Vet Radiol Ultrasound 47: 589-591, 2006.

15. Inampudi P, Jacobson JA, Fessell DP, et al: Soft-tissue lipomas: accuracy of sonography in diagnosis with pathologic correlation. Radiology 233: 763-767, 2004.

16. Munk PL, Lee MJ, Janzen DL, et al: Lipoma and liposarcoma: evaluation using CT and MR imaging. AJR Am J Roentgenol 169: 589-594, 1997.

17. Harrington AC, Adnot J and Chesser RS: Infiltrating lipomas of the upper extremities. J Dermatol Surg Oncol 16: 834-837, 1990.

18. Dionne GP and Seemayer TA: Infiltrating lipomas and angiolipomas revisited. Cancer 33: 732-738, 1974.

19. Simon HE and Senturia HR: Intermuscular lipoma of the thigh, with roentgenologic findings. South Med J 39: 624-626, 1946.

20. Meggitt BF and Wilson JN: The battered buttock syndrome - fat fractures. A report on a group of traumatic lipomata. Br J Surg 59: 165-169, 1972.

21. Higgs PE, Young VL, Schuster R and Weeks PM: Giant lipomas of the hand and forearm. South Med J 86: 887-890, 1993.

22. Echenique-Elizondo M: Intermuscular lipoma. J Am Coll Surg 193: 452, 2001.

23. Weiss SW: Lipomatous tumors. Monogr Pathol 38: 207-239, 1996. 